\title{
Hausdorff measure of noncompactness of matrix operators on some new difference sequence spaces
}

\author{
Elahe Abyar and Mohammad Bagher Ghaemi*
}

\section{"Correspondence:}

mghaemi@iust.ac.ir

Islamic Azad University, Karaj

Branch, Moazen, Alborz, Iran

\section{然 Springer}

\begin{abstract}
The new sequence spaces $X(r, s, t ; \Delta)$ for $X \in\left\{I_{\infty}, c, c_{0}\right\}$ have been defined by using generalized means and difference operator. In this work, we establish identities or estimates for the operator norms and the Hausdorff measure of noncompactness of certain matrix operators on some new difference sequence spaces $X(r, s, t ; \Delta)$ where $X \in\left\{I_{\infty}, C, C_{0}, I_{p}\right\}(1 \leq p<\infty)$, as derived by using generalized means. Further, we find the necessary and sufficient conditions for such operators to be compact by applying the Hausdorff measure of noncompactness. Finally, as applications we characterize some classes of compact operators between these new difference sequence spaces and some other BK-spaces.
\end{abstract}

Keywords: sequence space; difference operators; matrix transformation; generalized means; compact operators; Hausdorff measure of noncompactness

\section{Preliminaries and background}

The study of sequence spaces has been very useful in many branches of analysis. Recently, some new sequence spaces have been defined by using matrix domain of a suitable matrix. Beside this, the Hausdorff measure of noncompactness is very useful in the classification of compact operators between Banach spaces.

The difference sequence spaces were introduced for the first time by Kizmaz in [1]. Afterwards, many authors have introduced and studied some new sequence spaces defined by using the difference operator. For example, in [2] Ahmad and Mursaleen, in [3] Colak and Et, in [4] Basar and Altay, in [5] Orhan, in [6] Polat and Altay, in [7] Aydin and Basar, and in [8] Basar and Altay have introduced and studied some new sequence spaces defined by using a difference operator. Some authors like Malkowsky and Savas [9], Altay and Basar [10], Mursaleen and Noman [11] and Basarir and Kara [12] have defined the sequence spaces by using the generalized weighted means. For the first time, in 2011 Polat et al. [13] have introduced the new sequence spaces by combining both the weighted means and the difference operator. Then in 2015 Manna et al. [14] have introduced new sequence spaces defined by using both the generalized means and the difference operator.

In this paper we obtain some identities or estimates for the operator norms and the Hausdorff measure of noncompactness of certain matrix operators on new difference sequence spaces defined by Manna et al. Further, we find the necessary and sufficient condi-

(c) 2016 Abyar and Ghaemi. This article is distributed under the terms of the Creative Commons Attribution 4.0 International License (http://creativecommons.org/licenses/by/4.0/), which permits unrestricted use, distribution, and reproduction in any medium, provided you give appropriate credit to the original author(s) and the source, provide a link to the Creative Commons license, and indicate if changes were made. 
tions for such operators to be compact and we consider some matrix mappings between the spaces of the generalized means (these have been defined in [15]) and difference sequence spaces derived by using the generalized means. Finally, as applications we characterize some classes of compact operators between these new difference sequence spaces and some other $B K$-spaces.

In this section, we give some related definitions and preliminary results.

We shall write $w$ for the set of all complex sequences $x=\left(x_{k}\right)_{k=0}^{\infty}$. Any vector subspace of $w$ is called a sequence space. Let $l_{\infty}, c$, and $c_{0}$ denote the sets of all bounded, convergent and null sequences, respectively, and $l_{p}=\left\{x \in w: \sum_{k=0}^{\infty}\left|x_{k}\right|^{p}<\infty, 1 \leq p<\infty\right\}$. For an infinite matrix $A$ and a sequence space $X$, the matrix domain of $A$ is defined by $X_{A}=\{x \in w: A x \in X\}$. By $e$ and $e^{n}(n \in \mathbb{N})$, we denote the sequences such that $e_{k}=1$ for $k=0,1, \ldots$ and $e_{n}^{(n)}=1$ and $e_{k}^{n}=0(k \neq n)$. Let $b s$ and $c s$ be the sequence spaces of all bounded and convergent series, respectively. A sequence $\left(b_{n}\right)$ in a normed linear space $(X,\|\cdot\|)$ is called a Schauder basis for $X$ if for every $x \in X$, there is a unique sequence $\left(\delta_{n}\right)_{n=0}^{\infty}$ of scalars such that $x=\sum_{n=0}^{\infty} \delta_{n} b_{n}$. For definitions of $K$-space, $F K$-space, $B K$-space and $A K$-property we refer the reader to [16].

Throughout, the matrices are infinite matrices of complex number. Let $A=\left(a_{n k}\right)_{n, k}$ be an infinite matrix with real or complex entries $a_{n k}$. We write $A_{n}$ as the sequence of the $n$th row of $A$, that is, $A_{n}=\left(a_{n k}\right)_{k}$ for every $n$. In addition, if $x=\left(x_{k}\right) \in w$ then we define the $A$-transform of $x$ as the sequence $A x=\left(A_{n}(x)\right)_{n=0}^{\infty}$, that is,

$$
A_{n}(x)=\sum_{k=0}^{\infty} a_{n k} x_{k} \quad(n \in \mathbb{N}),
$$

provided the series on the right converges for each $n \in \mathbb{N}$. For any two sequence spaces $X$ and $Y$, we denote by $(X, Y)$ the class of all infinite matrices $A$ that map $X$ into $Y$. As is well known the $\beta$-dual a subset $X$ of $w$ is defined by

$$
X^{\beta}=\left\{a=\left(a_{k}\right) \in w: a x=\left(a_{k} x_{k}\right) \in c s \text { for all } x=\left(x_{k}\right) \in X\right\} .
$$

Thus $A \in(X, Y)$ if and only if $A_{n} \in X^{\beta}$ for all $n \in \mathbb{N}$ and $A x \in Y$ for all $x \in X$. An infinite matrix $T=\left(t_{n k}\right)$ is said to be triangle if $t_{n k}=0$ for $k>n$ and $t_{n n} \neq 0, n \in \mathbb{N}_{0}$ (where $\mathbb{N}_{0}=$ $\{0,1,2, \ldots\})$.

If $X \supset \phi$ is a $B K$-space and $a=\left(a_{n k}\right) \in w$, then we write

$$
\|a\|_{X}^{*}=\sup _{x \in S_{X}}\left|\sum_{k=0}^{\infty} a_{k} x_{k}\right|,
$$

provided the expression on the right is defined and finite which is the case whenever $a \in$ $X^{\beta}[17]$.

\section{The Hausdorff measure of noncompactness}

As is well known, one of the best methods in the characterization of compact operators between the Banach spaces depends on applying the Hausdorff measure of noncompactness. Therefore, many authors like Kara and Basarir in [18, 19], Basarir and Kara in [20, 21], Kara et al. in [22] and Alotaibi et al. in [23] characterized some classes of compact operators on the spaces in their papers by using the Hausdorff measure of noncompactness. 
Let $S$ and $M$ be subsets of a metric space $(X, d)$ and $\epsilon>0$. We say that $S$ is an $\epsilon$-net of $M$ if for every $x \in M$ there exists $s \in S$ such that $d(x, s)<\epsilon$. The $\epsilon$-net $S$ of $M$ is a finite $\epsilon$-net if $S$ is a finite set.

We denote by $M_{X}$ the collection of all bounded subsets of a metric space $(X, d)$. If $Q \in$ $M_{X}$, we denote by $\chi(Q)$ the Hausdorff measure of noncompactness of the set $Q$ and define it by

$$
\chi(Q)=\inf \{\epsilon>0: Q \text { has a finite } \epsilon \text {-net in } X\} .
$$

The function $\chi: M_{X} \rightarrow[0, \infty)$ is called the Hausdorff measure of noncompactness [24]. We can find the basic properties of the Hausdorff measure of noncompactness in [25].

We give an estimate for the Hausdorff measure of noncompactness in Banach spaces with a Schauder basis with the following theorem.

Theorem 2.1 ([25]) Let $X$ be a Banach space with a Schauder basis $\left(b_{k}\right)_{k=0}^{\infty}, P_{n}: X \rightarrow X$ $(n \in \mathbb{N})$ be the projector onto the linear span of $\left\{b_{0}, b_{1}, \ldots, b_{n}\right\}$ and $Q \in M_{X}$. Then we have

$$
\frac{1}{a} \cdot \lim \sup \left(\sup _{x \in Q}\left\|\left(I-P_{n}\right)(x)\right\|\right) \leq \chi(Q) \leq \limsup \left(\sup _{x \in Q}\left\|\left(I-P_{n}\right)(x)\right\|\right),
$$

where $a=\lim \sup _{n \rightarrow \infty}\left\|I-P_{n}\right\|$ and I denotes the identity operator on $X$.

Now, we show how to compute the Hausdorff measure of noncompactness in the spaces $c_{0}$ and $l_{p}(1 \leq p<\infty)$.

Theorem 2.2 ([26]) Let $Q$ be a bounded subset of the normed space $X$ and $X$ is $c_{0}$ or $l_{p}(1 \leq p<\infty)$. If $P_{n}: X \rightarrow X(n \in \mathbb{N})$ is the operator defined by $P_{n}(x)=x^{[n]}=$ $\left(x_{0}, x_{1}, \ldots, x_{n}, 0,0,0, \ldots\right)$ for all $x=\left(x_{k}\right)_{k=0}^{\infty} \in X$, then

$$
\chi(Q)=\lim _{n \rightarrow \infty}\left(\sup _{x \in Q}\left\|\left(I-P_{n}\right)(x)\right\|\right)
$$

We have the following result concerning with the Hausdorff measure of noncompactness in the matrix domain of triangles in normed sequence spaces.

Theorem 2.3 ([27]) Let $X$ be a normed sequence space, $T$ a triangle, and $\chi_{T}$ and $\chi$ denote the Hausdorff measure of noncompactness on $M_{X_{T}}$ and $M_{X}$. Then $\chi_{T}(Q)=\chi(T(Q))$ for all $Q \in M_{X_{T}}$.

Let $X$ and $Y$ be Banach spaces and $L \in B(X, Y)$. Then we denote by $\|L\|_{\chi}$ the Hausdorff measure of noncompactness of $L$ and define it by

$$
\|L\|_{\chi}=\chi\left(L\left(S_{X}\right)\right)=\chi\left(L\left(\bar{B}_{X}\right)\right)
$$

where $S_{X}=\{x \in X:\|x\|=1\}$ is the unit sphere and $\bar{B}_{X}=\{x \in X:\|x\| \leq 1\}$ is the unit ball in $X$, and 


\section{The sequence spaces $X(r, s, t ; \Delta)$ for $X \in\left\{I_{\infty}, c, c_{0}, I_{p}\right\}(1 \leq p<\infty)$}

In this subsection we first introduce the generalized means that given by Mursaleen and Noman in [15].

We first define the sets $\mho$ and $\mho_{0}$ as follows:

$$
\begin{aligned}
& \mho=\left\{u=\left(u_{n}\right)_{n=0}^{\infty} \in w: u_{n} \neq 0 \forall n\right\}, \\
& \mho_{0}=\left\{u=\left(u_{n}\right)_{n=0}^{\infty} \in w: u_{0} \neq 0\right\} .
\end{aligned}
$$

Let $r=\left(r_{n}\right) \in \mho, t=\left(t_{n}\right) \in \mho$, and $s=\left(s_{n}\right) \in \mho_{0}$. For any sequence $x=\left(x_{n}\right) \in w$, we define the sequence $y=\left(y_{n}\right)$ of generalized means of $x$ by:

$$
y_{n}=\frac{1}{r_{n}} \sum_{k=0}^{n} s_{n-k} t_{k} x_{k} \quad\left(n \in \mathbb{N}_{0}\right) .
$$

Further, we define the infinite matrix $A(r, s, t)$ of generalized means by

$$
\left(A(r, s, t)_{n k}\right)= \begin{cases}s_{n-k} t_{k} / r_{n}, & 0 \leq k \leq n \\ 0, & k>n\end{cases}
$$

for all $n, k \in \mathbb{N}$. By using the notation (1) and (3), we see that $y$ is the $A(r, s, t)$-transform of $x$, that is, $y=(A(r, s, t)) x$ for all $x \in w$. Let $D_{0}^{(s)}=\frac{1}{s_{0}}$ and

$$
D_{n}^{(s)}=\frac{1}{s_{0}^{n+1}}\left|\begin{array}{cccccc}
s_{1} & s_{0} & 0 & 0 & \cdots & 0 \\
s_{2} & s_{1} & s_{0} & 0 & \cdots & 0 \\
\vdots & \vdots & \vdots & \vdots & \cdots & \vdots \\
s_{n-1} & s_{n-2} & s_{n-3} & s_{n-4} & \cdots & s_{0} \\
s_{n} & s_{n-1} & s_{n-2} & s_{n-3} & \cdots & s_{1}
\end{array}\right| \quad(n=1,2,3, \ldots)
$$

so the inverse of $A(r, s, t)$ is the triangle $B=\left(b_{n k}\right)_{n k}$, which is defined by

$$
b_{n k}= \begin{cases}(-1)^{n-k} \frac{D_{n-k}^{(s)}}{t_{n}} r_{k}, & 0 \leq k \leq n, \\ 0, & k>n\end{cases}
$$

We now ready to introduce the sequence space $X(r, s, t ; \Delta)$ where $X \in\left\{l_{\infty}, c, c_{0}\right\}$ as the matrix domain of triangle $A(r, s, t ; \Delta)$ as follows:

$$
X(r, s, t ; \Delta)=\left\{x=\left(x_{n}\right) \in w:\left(\frac{1}{r_{n}} \sum_{k=0}^{n} s_{n-k} t_{k} \Delta x_{k}\right)_{n} \in X\right\},
$$

which is a combination of the generalized means and the difference operator $\Delta\left(\Delta x_{k}=x_{k}-\right.$ $\left.x_{k-1}, x_{-1}=0\right)$ and by using notation (1) we have $X(r, s, t ; \Delta)=X_{A(r, s, t ; \Delta)}$ where $A(r, s, t ; \Delta)=$ $A(r, s, t) \cdot \Delta$. It is clear that if $X$ is a sequence space, then $X(r, s, t ; \Delta)$ is a sequence space too and we call that the difference sequence space derived by using generalized means. 
Lemma 3.1 ([14]) The sequence spaces $X(r, s, t ; \Delta)$ where $X \in\left\{l_{\infty}, c, c_{0}\right\}$ are $B K$-spaces with the norm given by

$$
\begin{aligned}
\|x\|_{X(r, s, t ; \Delta)} & =\sup _{n}\left|\frac{1}{r_{n}} \sum_{k=0}^{n} s_{n-k} t_{k} \Delta x_{k}\right| \\
& =\sup _{n}\left|(A(r, s, t ; \Delta) x)_{n}\right|
\end{aligned}
$$

and so they are linearly isomorphic to the spaces $X \in\left\{l_{\infty}, c, c_{0}\right\}$, respectively.

We mention the following basic lemmas, which will be used in proving our results.

Lemma 3.2 Let $X$ be a BK-space with $A K$ or $X=l_{\infty}$ and $\overline{\Delta X}=X(r, s, t ; \Delta)$. If $a=\left(a_{k}\right) \in$ $(\overline{\Delta X})^{\beta}$, then $\tilde{a}=\left(\tilde{a}_{k}\right) \in X^{\beta}$ and we have

$$
\sum_{k=0}^{\infty} a_{k} x_{k}=\sum_{k=0}^{\infty} \tilde{a}_{k} y_{k}
$$

which holds for every $x=\left(x_{k}\right) \in X(r, s, t ; \Delta)$ with $y=A(r, s, t) \Delta x$ where

$$
\tilde{a}_{k}=\sum_{j=0}^{n} \sum_{k=0}^{n-j}(-1)^{k} \frac{D_{k}^{(s)}}{t_{k+j}} a_{j} r_{j} \quad(k \in \mathbb{N}) .
$$

Proof It is immediate by the same technique as ([11], Theorem 4.5).

Lemma 3.3 Let $X$ be a BK-space with $A K$ property or $X=l_{\infty}$ and $\overline{\Delta X}=X(r, s, t ; \Delta)$. Then we have

$$
\|a\|_{\frac{*}{\Delta X}}^{*}\|\tilde{a}\|_{X^{\beta}}
$$

for all $a=\left(a_{k}\right) \in(\overline{\Delta X})^{\beta}$, where $\tilde{a}=\left(\tilde{a}_{k}\right)$ is the sequence defined by $(7)$.

Proof Let $a=\left(a_{k}\right) \in(\overline{\Delta X})^{\beta}$. By applying Lemma 3.2 we have $\tilde{a}=\left(\tilde{a}_{k}\right) \in X^{\beta}$ and equality (6) holds for all sequences $x=\left(x_{k}\right) \in \overline{\Delta X}$ and $y=\left(y_{k}\right) \in X$. Further, it follows by the equality in Lemma 3.1 that $x \in S_{\overline{\Delta X}}$ if and only if $y \in S_{X}$. So, we derive from (2) and (6) that

$$
\begin{aligned}
\|a\|_{\Delta X}^{*} & =\sup _{x \in S_{\overline{\Delta X}}}\left|\sum_{k=0}^{\infty} a_{k} \Delta x_{k}\right| \\
& =\sup _{y \in S_{X}}\left|\sum_{k=0}^{\infty} \tilde{a}_{k} y_{k}\right| \\
& =\|\tilde{a}\|_{X^{\beta}} .
\end{aligned}
$$

This completes the proof.

Throughout this paper we assume $A=\left(a_{n k}\right)$ is an infinite matrix and $\tilde{A}=\left(\tilde{a}_{n k}\right)$ is the associated matrix which is defined by

$$
\tilde{a}_{n k}=\sum_{j=0}^{n} \sum_{k=0}^{n-j}(-1)^{k} \frac{D_{k}^{(s)}}{t_{k+j}} a_{n j} r_{j} \quad(n, k \in \mathbb{N})
$$


and provided the series on the right converge for all $n, k \in \mathbb{N}$. Then we have the following.

Lemma 3.4 Let $X$ be a $B K$-space with $A K$ property or $X=l_{\infty}$ and $\overline{\Delta X}=X(r, s, t ; \Delta), Y$ be any sequence space and $A=\left(a_{n k}\right)$ be an infinite matrix. If $A \in(\overline{\Delta X}, Y)$, then $\tilde{A} \in(X, Y)$ such that $A x=\tilde{A} y$ for all $x \in X$ and connected sequence $y=A(r, s, t) \cdot \Delta x$, where $\tilde{A}=\left(\tilde{a}_{n k}\right)$ is the associated matrix defined by (8).

Proof Let $x \in \overline{\Delta X}$ and $y \in Y$ be connected by equation (5) and $A \in(\overline{\Delta X}, Y)$. By applying Lemma 3.3, we have $\tilde{A}_{n} \in X^{\beta}$ for all $n \in \mathbb{N}$ and the equality $A x=\tilde{A} y$ holds, hence $\tilde{A} y \in Y$. Because every $y \in Y$ is the associated sequence of some $x \in \overline{\Delta X}$, we conclude that $\tilde{A} \in$ $(X, Y)$. This completes the proof.

Theorem 3.1 Let $\overline{\Delta X}=X(r, s, t ; \Delta)$ where $X$ be a $B K$-space with $A K$ or $X=l_{\infty}, A=\left(a_{n k}\right)$ is an infinite matrix and $\tilde{A}=\left(\tilde{a}_{n k}\right)$ is the associated matrix. If $A$ is in any of the classes $\left(\overline{\Delta X}, l_{\infty}\right),(\overline{\Delta X}, c)$, or $\left(\overline{\Delta X}, c_{0}\right)$, then we have

$$
\left\|L_{A}\right\|=\|A\|_{\left(\overline{\Delta X}, l_{\infty}\right)}=\sup _{n}\left\|\tilde{A}_{n}\right\|_{X}^{*}<\infty
$$

Proof By using Lemma 3.1 we know that the spaces $\overline{\Delta X}$ and $X$ are linearly isomorphic. Now by combining ([16], Remark 1.2(a)), and Lemma 3.3 the proof is complete.

Theorem 3.2 Let $X$ be a BK-space with $A K$ or $X=l_{\infty}$ and $\overline{\Delta X}=X(r, s, t ; \Delta)$. If $A$ is in $\left(\overline{\Delta X}, l_{1}\right)$, then

$$
\|A\|_{\left(\overline{\Delta X}, l_{1}\right)} \leq\left\|L_{A}\right\| \leq 4 \cdot\|A\|_{\left(\overline{\Delta X}, l_{1}\right)},
$$

where

$$
\|A\|_{\left(\overline{\Delta X}, l_{1}\right)}=\sup _{N \in F}\left\|\sum_{n \in N} \tilde{A}_{n}\right\|_{X}^{*}<\infty
$$

Proof By using Lemma 3.1 we know the spaces $\overline{\Delta X}$ and $X$ are linearly isomorphic. Now by combining ([16], Remark 1.2(b)) and Lemma 3.3 the proof is complete.

Theorem 3.3 Let $\overline{\Delta l_{1}}=l_{1}(r, s, t ; \Delta)$ and $1 \leq p<\infty$. If $A \in\left(\overline{\Delta l_{1}}, l_{p}\right)$, then

$$
\left\|L_{A}\right\|=\|A\|_{\left(\overline{\Delta l_{1}}, l_{p}\right)}=\sup _{k}\left(\sum_{n=0}^{\infty}\left|\tilde{a}_{n k}\right|^{p}\right)^{\frac{1}{p}}<\infty .
$$

Proof By using [14] and [11] we deduce $A \in\left(\overline{\Delta l_{1}}, l_{p}\right)$ if and only if the expression on the right of (8) converges and

$$
\sup _{k}\left(\sum_{n=0}^{\infty}\left|\tilde{a}_{n k}\right|^{p}\right)<\infty
$$


and

$$
\sup _{k}\left(\max _{0 \leq k \leq m}\left|a_{m k}^{(n)}\right|\right)<\infty \quad(\forall n \in \mathbb{N})
$$

where

$$
a_{m k}^{(n)}=\sum_{j=0}^{n} \sum_{k=0}^{n-j}(-1)^{k} \frac{D_{k}^{(s)}}{t_{k+j}} a_{n j} r_{j} \quad(0 \leq k \leq m, m \in \mathbb{N}) .
$$

By using Theorem 3.2, we immediate obtain the following result.

Corollary 3.1 Let $\overline{\Delta l_{p}}=l_{p}(r, s, t ; \Delta)(1 \leq p<\infty)$ and $A \in\left(\overline{\Delta l_{p}}, l_{1}\right)$. Then we have

$$
\|A\|_{\left(\overline{\Delta l_{p}}, l_{1}\right)}^{(r)} \leq\left\|L_{A}\right\| \leq 4 \cdot\|A\|_{\left(\overline{\Delta l_{p}}, l_{1}\right)}^{(r)},
$$

where

$$
\|A\| \frac{(r)}{\left(\overline{\Delta l}, l_{1}\right)}=\sup _{N \in F_{r}}\left(\sum_{k=0}^{\infty}\left|\sum_{n \in N} \tilde{a}_{n k}\right|^{q}\right)^{\frac{1}{q}} \quad(r \in \mathbb{N}) .
$$

Proof Let $X=l_{p}$ in Theorem 3.2.

Now, as a consequence of Corollary 3.1 we have the following lemma.

Lemma 3.5 Let $\overline{\Delta l_{1}}=l_{1}(r, s, t ; \Delta)$ and $1 \leq p<\infty$. If $A \in\left(l_{p}, \overline{\Delta l_{1}}\right)$, then

$$
\|A\|_{\left(l_{p}, \overline{\left.\Delta l_{1}\right)}\right.}^{(r)} \leq\left\|L_{A}\right\| \leq 4 \cdot\|A\|_{\left(l_{p}, \overline{\Delta l_{1}}\right)}^{(r)},
$$

where

$$
\|A\|_{\left(l_{p}, \overline{\left.\Delta l_{1}\right)}\right.}^{(r)}=\sup _{N \in F_{r}}\left(\sum_{k=0}^{\infty}\left|\sum_{n \in N} \tilde{a}_{n k}\right|^{q}\right)^{\frac{1}{q}} \quad(r \in \mathbb{N}),
$$

and $\tilde{a}_{n k}$ is defined by (8).

Proof Because the sequence spaces $\overline{\Delta l_{1}}$ and $l_{1}$ are norm-isomorphic,

$$
A \in\left(l_{p}, \overline{\Delta l_{1}}\right) \cong A \in\left(l_{p}, l_{1}\right) .
$$

Now this is a special case of ([16], Remark 1.2(b)) when $X=l_{p}$.

This completes the proof.

\section{Compact operators on the spaces $X(r, s, t ; \Delta)$ for $X \in\left\{I_{\infty}, c, c_{0}, I_{p}\right\}(1 \leq p<\infty)$}

In this section, we apply our results to obtaining some identities or estimates for the Hausdorff measure of noncompactness of certain matrix operators on the spaces $X(r, s, t ; \Delta)$ (where $X \in\left\{l_{\infty}, c, c_{0}, l_{p}\right\}(1 \leq p<\infty)$ ). Also, we consider the necessary and sufficient conditions for such operators to be compact. 
Lemma 4.1 ([28]) Let $X \supset \phi$ be a BK-space. Then we have:

(a) If $A \in\left(X, l_{\infty}\right)$, then

$$
0 \leq\left\|L_{A}\right\|_{\chi} \leq \limsup _{n \rightarrow \infty}\left\|A_{n}\right\|_{X}^{*} .
$$

(b) If $A \in\left(X, c_{0}\right)$, then

$$
\left\|L_{A}\right\|_{\chi}=\limsup _{n \rightarrow \infty}\left\|A_{n}\right\|_{X}^{*}
$$

(c) If $X$ has $A K$ or $X=l_{\infty}$ and $A \in(X, c)$, then

$$
\frac{1}{2} \cdot \limsup _{n \rightarrow \infty}\left\|A_{n}-\alpha\right\|_{X}^{*} \leq\left\|L_{A}\right\|_{\chi} \leq \limsup _{n \rightarrow \infty}\left\|A_{n}-\alpha\right\|_{X}^{*},
$$

where $\alpha=\left(\alpha_{k}\right)$ with $\alpha_{k}=\lim _{n \rightarrow \infty} a_{n k}$ for all $k \in \mathbb{N}$.

Now, by combining Lemmas $3.3,3.4$, and 4.1 we have the next result.

Theorem 4.1 Let $X$ be a BK-space with $A K$ or $X=l_{\infty}$ and $\overline{\Delta X}=X(r, s, t ; \Delta)$. Then we have:

(a) If $A \in\left(\overline{\Delta X}, l_{\infty}\right)$, then

$$
0 \leq\left\|L_{A}\right\|_{\chi} \leq \limsup _{n \rightarrow \infty}\left(\sum_{n}\left|\tilde{A}_{n}\right|\right)
$$

and

$$
L_{A} \text { is compact if } \lim _{n \rightarrow \infty}\left(\sum_{n}\left|\tilde{A}_{n}\right|\right)=0 .
$$

(b) If $A \in\left(\overline{\Delta X}, c_{0}\right)$, then

$$
\left\|L_{A}\right\|_{\chi}=\limsup _{n \rightarrow \infty}\left(\sum_{n}\left|\tilde{A}_{n}\right|\right),
$$

and

$$
L_{A} \text { is compact if and only if } \lim _{n \rightarrow \infty}\left(\sum_{n}\left|\tilde{A}_{n}\right|\right)=0 .
$$

(c) If $A \in(\overline{\Delta X}, c)$, then

$$
\frac{1}{2} \cdot \limsup _{n \rightarrow \infty}\left(\sum_{n}\left|\tilde{A}_{n}-\tilde{\alpha}\right|\right) \leq\left\|L_{A}\right\|_{\chi} \leq \limsup _{n \rightarrow \infty}\left(\sum_{n}\left|\tilde{A}_{n}-\tilde{\alpha}\right|\right)
$$

and

$$
L_{A} \text { is compact if and only if } \lim _{n \rightarrow \infty}\left(\sum_{n}\left|\tilde{A}_{n}-\tilde{\alpha}\right|\right)=0,
$$

where $\tilde{\alpha}=\left(\tilde{\alpha}_{k}\right)$ with $\tilde{\alpha}_{k}=\lim _{n \rightarrow \infty} \tilde{a}_{n k}$ for all $k \in \mathbb{N}$. 
Proof It is clear that (10), (12), and (14) are, respectively, obtained from (9), (11), and (13) by using (4). So, to prove (9), (11), and (13) we have the following.

Because $\overline{\Delta X}$ is a $B K$-space, hence by combining Lemma 3.3 and parts (a) and (b) of Lemma 4.1 we obtain (9) and (11).

To prove (13), if $A \in(\overline{\Delta X}, c)$, then by Lemma 3.4 we have $\tilde{A} \in(X, c)$. So, by part (c) of Lemma 4.1 we have

$$
\frac{1}{2} \cdot \limsup _{n \rightarrow \infty}\left(\sum_{n}\left|\tilde{A}_{n}-\tilde{\alpha}\right|\right) \leq\left\|L_{\tilde{A}}\right\|_{\chi} \leq \limsup _{n \rightarrow \infty}\left(\sum_{n}\left|\tilde{A}_{n}-\tilde{\alpha}\right|\right)
$$

where $\tilde{\alpha}=\left(\tilde{\alpha}_{k}\right)$ and $\tilde{\alpha}_{k}=\lim _{n \rightarrow \infty} \tilde{a}_{n k}$ for all $k \in \mathbb{N}$. Now, we write $S=S_{X}$ and $\overline{\Delta S}=S_{\overline{\Delta X}}$ for short. Then we obtain by (3) and ([16], Remark 1.2(c))

$$
\left\|L_{A}\right\|_{\chi}=\chi\left(L_{A}(\overline{\Delta S})\right)=\chi(A \overline{\Delta S})
$$

and

$$
\left\|L_{\tilde{A}}\right\|_{\chi}=\chi\left(L_{\tilde{A}}(S)\right)=\chi(\tilde{A} S) .
$$

Also, by using the equality in Lemma 3.1 we have $x \in \overline{\Delta S}$ if and only if $y \in S$, and since $A x=\tilde{A} y$ by Lemma 3.4, we derive that $A \overline{\Delta S}=\tilde{A} S$. This result together (16) and (17) leads to $\left\|L_{A}\right\|_{\chi}=\left\|L_{\tilde{A}}\right\|_{\chi}$. So, we get (13) from (15). This completes the proof.

The following example will show that it is possible for $L_{A}$ in (10) to be compact but $\lim _{n \rightarrow \infty}\left(\sum_{n}\left|\tilde{A}_{n}\right|\right) \neq 0$. Hence, in general, we have just 'if' in (10) of Theorem 4.1(a).

Example 4.1 Let $\overline{\Delta X}=X(r, s, t ; \Delta)$ where $X \in\left\{l_{\infty}, c, c_{0}\right\}$. Then let for $n=1$ the sequence $s=\left(s_{n}\right)=0$ and for every $n \neq 1, s=\left(s_{n}\right)=1$. Also, let the sequences $r=\left(r_{n}\right)=1, t=\left(t_{n}\right)=1$ are constant. Define the matrix $A=\left(a_{n k}\right)$ by

$$
a_{n k}= \begin{cases}\frac{s_{n-k} t_{k}}{r_{n}} & (0 \leq k \leq 2), \\ 0 & (k \geq 3) .\end{cases}
$$

Then, for every $x=\left(x_{n}\right) \in \overline{\Delta X}$, we have $A x=\left[\frac{s_{2} t_{0}}{r_{2}} x_{0} e^{(0)}+\frac{s_{1} t_{1}}{r_{2}} x_{1} e^{(1)}+\frac{s_{0} t_{2}}{r_{2}} x_{2} e^{(2)}\right]$. So $A \in$ $\left(\overline{\Delta X}, l_{\infty}\right)$. It is clear that $L_{A}$ is of finite rank so is compact. By using (8) we obtain $\tilde{A}_{n}=1$, for all $n \in \mathbb{N}$. Thus, we have that $\sum_{n}\left|\tilde{A}_{n}\right|=1$ for all $n \in \mathbb{N}$, which implies that $\lim _{n \rightarrow \infty} \sum_{n}\left|\tilde{A}_{n}\right|=1 \neq 0$.

Let $F_{r}(r \in \mathbb{N})$ be the subcollection of $F$ consisting of all nonempty and finite subsets of $\mathbb{N}$ with elements that are greater than $r$, that is,

$$
F_{r}=\{N \in F: n>r \text { for all } n \in N\} \quad(r \in \mathbb{N}) .
$$

Lemma 4.2 ([11]) Let $X \supset \phi$ be a $B K$-space. If $A \in\left(X, l_{1}\right)$, then

$$
\lim _{r \rightarrow \infty}\left(\sup _{N \in F_{r}}\left\|\sum_{n \in N} A_{n}\right\|_{X}^{*}\right) \leq\left\|L_{A}\right\|_{\chi} \leq 4 \cdot \lim _{r \rightarrow \infty}\left(\sup _{N \in F_{r}}\left\|\sum_{n \in N} A_{n}\right\|_{X}^{*}\right) .
$$


By using Lemma 4.2, we have the next result.

Theorem 4.2 Let $X$ be a BK-space with $A K$ and $\overline{\Delta X}=X(r, s, t ; \Delta)$. If $A \in\left(\overline{\Delta X}, l_{1}\right)$, then

$$
\lim _{r \rightarrow \infty}\|A\|_{\left(\overline{\Delta X}, l_{1}\right)}^{(r)} \leq\left\|L_{A}\right\|_{\chi} \leq 4 \cdot \lim _{r \rightarrow \infty}\|A\|_{\left(\overline{\Delta X}, l_{1}\right)}^{(r)},
$$

where

$$
\|A\|_{\left(\overline{\Delta X}, l_{1}\right)}^{(r)}=\sup _{N \in F_{r}}\left\|\sum_{n \in N} \tilde{A}_{n}\right\|_{X}^{*} \quad(r \in \mathbb{N})
$$

and

$$
L_{A} \text { is compact if and only if } \lim _{r \rightarrow \infty}\|A\|_{\left(\overline{\Delta X}, l_{1}\right)}^{(r)}=0 \text {. }
$$

Proof By combining Lemma 3.3 and Lemma 4.2 we obtain (18). By using (4), we get (19) from (18).

By using the previous results we have the next result.

Theorem 4.3 Let $\overline{\Delta l_{1}}=l_{1}(r, s, t ; \Delta)$ and $1 \leq p \leq \infty$. If $\left.A \in \overline{\left(\Delta l_{1}\right.}, l_{p}\right)$, then

$$
\left\|L_{A}\right\|_{\chi}=\lim _{r \rightarrow \infty}\left(\sup _{k}\left(\sum_{n=r}^{\infty}\left|\tilde{a}_{n k}\right|^{p}\right)^{\frac{1}{p}}\right)
$$

and

$$
L_{A} \text { is compact if and only if } \lim _{r \rightarrow \infty}\left(\sup _{k}\left(\sum_{n=r}^{\infty}\left|\tilde{a}_{n k}\right|^{p}\right)^{\frac{1}{p}}\right)=0 \text {. }
$$

Proof It can be similarly proved by the same technique as Theorem 3.9 in [15].

In the following example we show that there exist matrix operators in the class $B\left(\overline{\Delta l_{1}}, l_{p}\right)$ which are not compact $(1 \leq p<\infty)$, that is, the limit in (20) may not be zero.

Example 4.2 Let $A=\left(a_{n k}\right)$ be the infinite matrix defined by

$$
a_{n k}= \begin{cases}s_{n-k} t_{k} / r_{n}, & 0 \leq k \leq 1 \\ 0, & k>2\end{cases}
$$

where $s=\left(s_{n}\right)=1$ if $n=0$ and $s=\left(s_{n}\right)=0$ for every $n \neq 0$ and $r=\left(r_{n}\right)=1=t=\left(t_{n}\right)$. Then $A \in\left(\overline{\Delta l_{1}}, l_{1}\right)$ because $\overline{\Delta l_{1}}$ is the matrix domain of $A$ in $l_{1}$ and hence $A \in\left(\overline{\Delta l_{1}}, l_{p}\right)(1 \leq p<\infty)$. Further, it is easy to show that the associated matrix $\tilde{A}$ is the identity matrix, that is, $\tilde{a}_{n n}=1$ and $\tilde{a}_{n k}=0$ for $k \neq n(n \in \mathbb{N})$. Now, let $r \in \mathbb{N}$ be given. Then we have, for every $k \in \mathbb{N}$,

$$
\sum_{n=r}^{\infty}\left|\tilde{a}_{n k}\right|^{p}= \begin{cases}1, & k \geq r \\ 0, & k<r\end{cases}
$$


This implies that

$$
\sup _{k}\left(\sum_{n=r}^{\infty}\left|\tilde{a}_{n k}\right|^{p}\right)^{\frac{1}{p}}=1 \quad(r \in \mathbb{N}),
$$

which shows that by (20) that $\left\|L_{A}\right\|_{\chi}=1 \neq 0$ and hence $L_{A}$ is not compact.

By using Lemmas 3.5 and 4.2 we have the following result on the Hausdorff measure of noncompactness.

Corollary 4.1 Let $\overline{\Delta l_{1}}=l_{1}(r, s, t ; \Delta)$ and $1 \leq p \leq \infty$. If $A \in\left(l_{p}, \overline{\Delta l_{1}}\right)$, then

$$
\|A\|_{\left(l_{p}, \overline{\left.\Delta l_{1}\right)}\right.}^{(r)} \leq\left\|L_{A}\right\|_{\chi} \leq 4 \cdot\|A\|_{\left(l_{p}, \overline{\Delta l_{1}}\right)}^{(r)},
$$

where

$$
\|A\|_{\left(l_{p}, \overline{\Delta l_{1}}\right)}^{(r)}=\sup _{k}\left(\sum_{n=r}^{\infty}\left|\tilde{a}_{n k}\right|^{q}\right)^{\frac{1}{q}} \quad(r \in \mathbb{N})
$$

and

$$
L_{A} \text { is compact if and only if } \lim _{r \rightarrow \infty}\|A\|_{\left(l_{p}, \overline{\left.\Delta l_{1}\right)}\right.}^{(r)}=0 \text {. }
$$

Proof We know the spaces $l_{1}$ and $\overline{\Delta l_{1}}$ are norm-isomorphic. So, the proof is complete by Lemmas 3.5 and 4.2 .

\section{Some applications}

In this section, by applying our results in the previous sections we consider some relations between the spaces of generalized means and difference sequence spaces derived by using the generalized means and we characterize some classes of compact operators between these new difference sequence spaces and some other $B K$-spaces. Also, we derive some identities and estimates for the operator norms and the Hausdorff measure of noncompactness on these spaces.

Throughout, $X(r, s, t)$ is the sequence space of the generalized means and for more details we refer the reader to [11]; further, for an arbitrary $B K$-space $X$, we put $\bar{X}=X(r, s, t)$, and $\overline{\Delta X}=X(r, s, t ; \Delta)$.

The first result is a consequence of Theorem 3.1 and Theorem 4.1.

Corollary 5.1 Let $X$ be a $B K$-space with $A K$ or $X=l_{\infty}$. Then:

If $A$ is in any of the classes $\left(\overline{\Delta X}, \overline{l_{\infty}}\right),(\overline{\Delta X}, \bar{c})$, or $\left(\overline{\Delta X}, \overline{c_{0}}\right)$ then

$$
\left\|L_{A}\right\|=\|A\|_{\left(\overline{\Delta X}, \overline{l_{\infty}}\right)}=\sup _{n}\left\|\tilde{A}_{n}\right\|_{X}^{*}<\infty
$$

where $\tilde{A}_{n}=\left(\tilde{a}_{n k}\right)$ is the associated matrix defined by

$$
\tilde{a}_{n k}=\sum_{j=k}^{\infty}(-1)^{j-k} D_{j-k}^{(s)} r_{k} a_{n j} / t_{j} \quad(n, k \in \mathbb{N}) .
$$




\section{Further:}

(a) If $A \in\left(\overline{\Delta X}, \overline{l_{\infty}}\right)$, then

$$
0 \leq\left\|L_{A}\right\|_{\chi} \leq \limsup _{n \rightarrow \infty}\left\|\tilde{A}_{n}\right\|_{X}^{*}
$$

and

$$
L_{A} \text { is compact if } \lim _{n \rightarrow \infty}\left\|\tilde{A}_{n}\right\|_{X}^{*}=0 \text {. }
$$

(b) If $A \in(\overline{\Delta X}, \bar{c})$, then

$$
\frac{1}{2} \cdot \limsup _{n \rightarrow \infty}\left\|\tilde{A}_{n}-\tilde{\alpha}\right\|_{X}^{*} \leq\left\|L_{A}\right\|_{\chi} \leq \limsup _{n \rightarrow \infty}\left\|\tilde{A}_{n}-\tilde{\alpha}\right\|_{X}^{*}
$$

and

$$
L_{A} \text { is compact if and only if } \lim _{n \rightarrow \infty}\left\|\tilde{A}_{n}-\tilde{\alpha}\right\|_{X}^{*}=0 \text {, }
$$

where $\tilde{\alpha}=\left(\tilde{\alpha}_{k}\right)$ with $\tilde{\alpha}_{k}=\lim _{n \rightarrow \infty} \tilde{a}_{n k}$ for all $k \in \mathbb{N}$.

(c) If $A \in\left(\overline{\Delta X}, \overline{c_{0}}\right)$, then

$$
\left\|L_{A}\right\|_{\chi}=\limsup _{n \rightarrow \infty}\left\|\tilde{A}_{n}\right\|_{X}^{*}
$$

and

$$
L_{A} \text { is compact if and only if } \lim _{n \rightarrow \infty}\left\|\tilde{A}_{n}\right\|_{X}^{*}=0 \text {. }
$$

Proof We know that the spaces $\overline{l_{\infty}}, \bar{c}, \overline{c_{0}}$ and $l_{\infty}, c, c_{0}$ are norm-isomorphic, respectively. Now by Theorems 3.1 and 4.1 the proof is completed.

The next result is a consequence of Theorem 3.2 and Theorem 4.2.

Corollary 5.2 Let $X$ be a $B K$-space with $A K$ or $X=l_{\infty}$. If $A \in\left(\overline{\Delta X}, \overline{l_{1}}\right)$, then

$$
\|A\|_{\left(\overline{\Delta X}, \overline{l_{1}}\right)} \leq\left\|L_{A}\right\| \leq 4 \cdot\|A\|_{\left(\overline{\Delta X}, \overline{l_{1}}\right)},
$$

where

$$
\|A\|_{\left(\overline{\Delta X}, \bar{l}_{1}\right)}=\sup _{N \in F}\left\|\sum_{n \in N} \tilde{A}_{n}\right\|_{X}^{*}<\infty
$$

and $\tilde{A}_{n}=\left(\tilde{a}_{n k}\right)$ is similarly defined by (24).

Further:

If $A \in\left(\overline{\Delta X}, \overline{l_{1}}\right)$, then

$$
\|A\|_{\left(\overline{\Delta X}, \overline{l_{1}}\right)}^{(r)} \leq\left\|L_{A}\right\|_{\chi} \leq 4 \cdot\|A\|_{\left(\overline{\Delta X}, \overline{l_{1}}\right)}^{(r)},
$$


where

$$
\|A\|_{\left(\overline{\Delta X}, \overline{l_{1}}\right)}^{(r)}=\sup _{N \in F_{r}}\left\|\sum \tilde{A}_{n}\right\|_{X}^{*}<\infty
$$

and

$$
L_{A} \text { is compact if and only if } \lim _{r \rightarrow \infty}\|A\|_{\left(\overline{\Delta X}, \overline{l_{1}}\right)}^{(r)}=0 \text {. }
$$

Proof We know that the spaces $\overline{l_{1}}$ and $l_{1}$ are norm-isomorphic. Now by Theorems 3.2 and 4.2 the proof is complete.

Now we have a special case of Theorem 3.3 in [28] in the new spaces $\overline{\Delta X}$ where $X \in$ $\left\{l_{\infty}, c, c_{0}\right\}$.

Corollary 5.3 Let $X$ be a BK-space with $A K$ or $X=l_{\infty}$ and $\bar{Y}$ denote any of the spaces $\overline{l_{\infty}}$, $\bar{c}$, or $\overline{c_{0}}$ we and let $A$ be an infinite matrix. Then:

If $A \in(\bar{Y}, \overline{\Delta X})$ then we have

$$
\left\|L_{A}\right\|=\|A\|_{(\bar{Y}, \overline{\Delta l \infty})}=\sup _{n}\left(\sum_{k=0}^{\infty}\left|\tilde{a}_{n k}\right|\right)<\infty,
$$

where $\tilde{A}_{n}=\left(\tilde{a}_{n k}\right)$ is the associated matrix defined by (8).

Further:

(a) If $A \in\left(\overline{l_{\infty}}, \overline{\Delta l_{\infty}}\right)$, then

$$
0 \leq\left\|L_{A}\right\|_{\chi} \leq \limsup _{n \rightarrow \infty}\left(\sum_{k=0}^{\infty}\left|\tilde{a}_{n k}\right|\right)
$$

and

$$
L_{A} \text { is compact if } \lim _{n \rightarrow \infty}\left(\sum_{k=0}^{\infty}\left|\tilde{a}_{n k}\right|\right)=0 .
$$

(b) If $A \in\left(\bar{c}, \overline{\Delta l_{\infty}}\right)$, then

$$
\frac{1}{2} \cdot \limsup _{n \rightarrow \infty}\left(\sum_{k=0}^{\infty}\left|\tilde{a}_{n k}-\tilde{\alpha}_{k}\right|\right) \leq\left\|L_{A}\right\|_{\chi} \leq \limsup _{n \rightarrow \infty}\left(\sum_{k=0}^{\infty}\left|\tilde{a}_{n k}-\tilde{\alpha}_{k}\right|\right)
$$

and

$$
L_{A} \text { is compact if and only if } \lim _{n \rightarrow \infty}\left(\sum_{k=0}^{\infty}\left|\tilde{a}_{n k}-\tilde{\alpha}_{k}\right|\right)=0,
$$

where $\tilde{\alpha}=\left(\tilde{\alpha}_{k}\right)$ with $\tilde{\alpha}_{k}=\lim _{n \rightarrow \infty} \tilde{a}_{n k}$ for all $k \in \mathbb{N}$.

(c) If $A \in\left(\bar{c}, \overline{\Delta l_{\infty}}\right)$, then

$$
\left\|L_{A}\right\|_{\chi}=\limsup _{n \rightarrow \infty}\left(\sum_{k=0}^{\infty}\left|\tilde{a}_{n k}\right|\right)
$$


and

$$
L_{A} \text { is compact if and only if } \lim _{n \rightarrow \infty}\left(\sum_{k=0}^{\infty}\left|\tilde{a}_{n k}\right|\right)=0
$$

The spaces $b s, c s$, and $c s_{0}$ are $B K$-spaces with the same norm given by $\|x\|_{b s}=$ $\sup _{n}\left|\sum_{k=0}^{n} x_{k}\right|$. Now, by using Theorems 3.1, 4.1 and 3.2, 4.2 we derive the next consequence.

Corollary 5.4 Let $X \supset \phi$ be a BK-space and $A$ an infinite matrix. If $A$ is in any of the classes $\left(\overline{\Delta X}, c s_{0}\right),(\overline{\Delta X}, c s)$, or $(\overline{\Delta X}, b s)$, then

$$
\left\|L_{A}\right\|=\sup _{n}\|\tilde{A}\|_{(\overline{\Delta X}, b s)}^{(n)}<\infty
$$

where

$$
\|\tilde{A}\|_{(\overline{\Delta X}, b s)}^{(n)}=\sup _{n}\left(\sum_{k=0}^{\infty}\left|\sum_{m=0}^{n} \tilde{a}_{m k}\right|\right) \quad(n \in \mathbb{N}) .
$$

Furthermore, we have:

(a) If $A \in\left(\overline{\Delta X}, c s_{0}\right)$, then

$$
\left\|L_{A}\right\|_{\chi}=\limsup _{n \rightarrow \infty}\|\tilde{A}\|_{(\overline{\Delta X}, b s)}^{(n)}
$$

and

$$
L_{A} \text { is compact if and only if } \lim _{n \rightarrow \infty}\|\tilde{A}\|_{(\overline{\Delta X}, b s)}^{(n)}=0 \text {. }
$$

(b) If $A \in(\overline{\Delta X}, b s)$, then

$$
0 \leq\left\|L_{A}\right\|_{\chi} \leq \limsup _{n \rightarrow \infty}\|\tilde{A}\|_{(\overline{\Delta X}, b s)}^{(n)}
$$

and

$$
L_{A} \text { is compact if } \lim _{n \rightarrow \infty}\|\tilde{A}\|_{\left(\frac{n)}{\Delta X}, b s\right)}^{(n)}=0 \text {. }
$$

(c) If $X$ has $A K$ and $A \in(\overline{\Delta X}, c s)$, then

$$
\frac{1}{2} \cdot \limsup _{n \rightarrow \infty}\|\tilde{A}-\tilde{\alpha}\|_{(\overline{\Delta X}, b s)}^{(n)} \leq\left\|L_{A}\right\|_{\chi} \leq \limsup _{n \rightarrow \infty}\|\tilde{A}-\tilde{\alpha}\|_{(\overline{\Delta X}, b s)}^{(n)}
$$

where $\tilde{\alpha}=\left(\tilde{\alpha}_{k}\right)$ with $\tilde{\alpha}_{k}=\lim _{m \rightarrow \infty} \tilde{a}_{m k}$ for all $k \in \mathbb{N}$ and

$$
L_{A} \text { is compact if and only if } \lim _{n \rightarrow \infty} \limsup _{n \rightarrow \infty}\|\tilde{A}-\tilde{\alpha}\|_{(\overline{\Delta X}, b s)}^{(n)}=0 \text {. }
$$

Proof The proof is quite similar to Corollary 5.1 in [16] for the new spaces $\overline{\Delta X}$ where $X \in\left\{l_{\infty}, c, c_{0}\right\}$. 
We denote the space of all sequences of bounded variation by $b v$ and define

$$
b v=\left\{x=\left(x_{k}\right) \in w:\left(x_{k}-x_{k-1}\right) \in l_{1}\right\} .
$$

So, the space $b v$ is a $B K$-space with

$$
\|x\|_{b v}=\sum_{k=0}^{\infty}\left|x_{k}-x_{k-1}\right| .
$$

Now, we have the following result.

Corollary 5.5 Let $X \supset \phi$ be a $B K$-space. If $A \in(\overline{\Delta X}, b v)$, then

$$
\|A\|_{(\overline{\Delta X}, b v)} \leq\left\|L_{A}\right\| \leq 4 \cdot\|A\|_{(\overline{\Delta X}, b v)},
$$

where

$$
\|A\|_{(\overline{\Delta X}, b v)}=\sup _{N \in F}\left(\sum_{n \in N}\left|\sum_{k=0}^{n}\left(\tilde{A}_{k}-\tilde{A}_{k-1}\right)\right|\right)<\infty .
$$

\section{Furthermore:}

If $A \in(\overline{\Delta X}, b v)$, then

$$
\|A\|_{(\overline{\Delta X}, b v)}^{(r)} \leq\left\|L_{A}\right\|_{X} \leq 4 \cdot\|A\|_{(\overline{\Delta X}, b v)}^{(r)}
$$

where

$$
\|A\|_{(\overline{\Delta X}, b \nu)}^{(r)}=\sup _{N \in F_{r}}\left(\sum_{n \in N}\left|\sum_{k=0}^{n}\left(\tilde{A}_{k}-\tilde{A}_{k-1}\right)\right|\right)<\infty \quad(r \in \mathbb{N})
$$

and

$$
L_{A} \text { is compact if and only if } \lim _{r \rightarrow \infty}\|A\|_{(\overline{\Delta X}, b v)}^{(r)}=0 .
$$

Proof We proceed by Theorem 4.4 in [28] and Theorem 4.2.

We denote $b v^{p}$ for the space of all sequences of $p$-bounded variation and define it by

$$
b v^{p}=\left\{x=\left(x_{k}\right) \in w:\left(x_{k}-x_{k-1}\right) \in l_{p}\right\} \quad(1<p<\infty) .
$$

$b v^{p}$ is a $B K$-space with its natural norm (cf. [17]). For every $a=\left(a_{k}\right) \in\left(b v^{p}\right)^{\beta}$, we have

$$
\|a\|_{b v^{p}}^{*}=\left(\sum_{k=0}^{\infty}\left|\sum_{j=k}^{\infty} a_{j}\right|^{q}\right)^{\frac{1}{q}} .
$$

Corollary 5.6 Let $X$ denote any of the spaces $l_{\infty}$ or $c_{0}$, and let $A$ be an infinite matrix, $1<p<\infty$, and $q=p /(p-1)$. If $A \in\left(b v^{p}, \overline{\Delta X}\right)$, then

$$
\left\|L_{A}\right\|=\|A\|_{\left(b v^{p}, \overline{\left.\Delta l_{\infty}\right)}\right.}^{(r)}=\sup _{n>r}\left(\sum_{k=0}^{\infty}\left|\sum_{j=k}^{\infty} \tilde{a}_{n j}\right|^{q}\right)^{\frac{1}{q}} \quad(r \in \mathbb{N}) .
$$




\section{Further:}

(a) If $A \in\left(b v^{p}, \overline{\Delta l_{\infty}}\right)$, then

$$
0 \leq\left\|L_{A}\right\|_{\chi} \leq \lim _{r \rightarrow \infty}\|A\|_{\left(b v^{p}, \overline{\left.\Delta l_{\infty}\right)}\right.}^{(r)}
$$

and

$$
L_{A} \text { is compact if } \lim _{r \rightarrow \infty}\|A\|_{\left(b v^{p}, \overline{\left.\Delta l_{\infty}\right)}\right.}^{(r)}=0 .
$$

(b) If $A \in\left(b v^{p}, \overline{\Delta c_{0}}\right)$, then

$$
\left\|L_{A}\right\|_{\chi}=\lim _{r \rightarrow \infty}\|A\|_{\left(b v v^{p}, \overline{\left.\Delta l_{\infty}\right)}\right.}^{(r)}
$$

and

$$
L_{A} \text { is compact if and only if } \lim _{r \rightarrow \infty}\|A\|_{\left(b v^{p}, \overline{\left.\Delta l_{\infty}\right)}\right.}^{(r)}=0 \text {. }
$$

Proof The proof is a special case of ([28], Theorem 3.3) when $X=b v^{p}$. Then this completes the proof Lemma 3.1.

\section{Competing interests}

The authors declare that they have no competing interests.

\section{Authors' contributions}

All authors of the manuscript have read and agreed to its content and are accountable for all aspects of the accuracy and integrity of the manuscript in accordance with ICMJE criteria.

\section{Received: 24 May 2016 Accepted: 12 October 2016 Published online: 25 October 2016}

\section{References}

1. Kizmaz, H: On certain sequence spaces. Can. Math. Bull. 24(2), 169-176 (1981)

2. Ahmad, ZU, Mursaleen, M: Kothe-Toeplitz duals of some new sequence spaces and their matrix maps. Publ. Inst. Math. (Belgr.) 42(56), 57-61 (1987)

3. Colak, R, Et, M: On some generalized difference sequence spaces and related matrix transformations. Hokkaido Math. J. 26(3), 483-492 (1997)

4. Altay, B, Basar, F: The fine spectrum and matrix domain of the difference operator $\Delta$ on the sequence space $/(p)$ $(0<p<1)$. Commun. Math. Anal. 2(2), 1-11 (2007)

5. Orhan, C: Matrix transformations on Cesaro difference sequence spaces. Commun. Fac. Sci. Univ. Ank. Sér. A1 Math. 33(1), 1-8 (1984)

6. Polat, H, Atay, B: On some new Euler difference sequence spaces. Southeast Asian Bull. Math. 30, $209-220$ (2006)

7. Aydin, C, Basar, F: Some new difference sequence spaces. Appl. Math. Comput. 157(3), 677-693 (2004)

8. Basar, F, Altay, B: On the space of sequences of $p$-bounded variation and related matrix mappings. Ukr. Math. J. 55(1), 136-147 (2003)

9. Malkowsky, E, Savas, E: Matrix transformations between sequence spaces of generalized weighted means. Appl. Math. Comput. 147, 333-345 (2004)

10. Altay, B, Basar, F: Generalization of the sequence space /(p) derived by weighted mean. J. Math. Anal. Appl. 330, 174-185 (2007)

11. Mursaleen, M, Noman, AK: On generalized means and some related sequence spaces. Comput. Math. Appl. 61(4), 988-999 (2011)

12. Basarir, M, Kara, EE: On the $m$ th order difference sequence space of generalized weighted mean and compact operators. Acta Math. Sci. 33(3), 797-813 (2013)

13. Polat, H, Karakaya, V, Simsek, N: Difference sequence spaces derived by using a generalized weighted mean. Appl. Math. Lett. 24(5), 608-614 (2011)

14. Manna, A, Maji, A, Srivastava, PD: Difference sequence spaces derived by using generalized means. J. Egypt. Math. Soc. 23, 127-133 (2015)

15. Mursaleen, M, Noman, AK: Hausdorff measure of noncompactness of certain matrix operators on the sequence spaces of generalized means. J. Math. Anal. 417, 96-111 (2014)

16. Abyar, E, Ghaemi, MB: Hausdorff measure of noncompactness of matrix operators on some sequence spaces of a double sequential band matrix. J. Inequal. Appl. 2015, 406 (2015) 
17. Malkowsky, E, Rakocevic, V, Zivkovic, S: Matrix transformations between the sequence space $b v^{p}$ and certain BK-spaces. Bull. Cl. Sci. Math. Nat. Sci. Math. 123(27), 33-46 (2002)

18. Kara, EE, Basarir, M: On compact operators and some Euler $B^{(m)}$-difference sequence spaces. J. Math. Anal. Appl. 279(2), 459-511 (2011)

19. Kara, EE, Basarir, M: On compact operators and some Euler $B^{(m)}$-difference sequence spaces. J. Math. Anal. Appl. 379, 499-511 (2011)

20. Basarir, M, Kara, EE: On compact operators on the Riesz $B^{(m)}$-difference sequence space. Iran. J. Sci. Technol. 35(A4), 279-285 (2011)

21. Basarir, M, Kara, EE: On compact operators on the Riesz $B^{(m)}$-difference sequence space II. Iran. J. Sci. Technol. 36(A3), 271-276 (2012) (Special issue - Mathematics)

22. Kara, EE, Basarir, M, Mursaleen, M: Compactness of matrix operators on some sequence spaces derived by Fibonacci numbers. Kragujev. J. Math. 39(2), 217-230 (2015)

23. Alotaibi, A, Mursaleen, M, Mohiuddine, SA: Compact matrix operators on a new sequence space related to $I_{p}$ spaces. J. Inequal. Appl. 2016, 193 (2016)

24. Malkowsky, E, Rakocevic, V, Zivkovic, S: Matrix transformations between the sequence spaces $W_{0}^{(p)}(\Lambda), V_{0}^{(p)}(\Lambda), C_{0}^{(p)}(\Lambda)$ $(1<p<\infty)$ and certain BK-spaces. Appl. Math. Comput. 147(2), 377-396 (2004)

25. Malkowsky, E: Modern functional analysis in the theory of sequence spaces and matrix transformations. Jordan J. Math. Stat. 1(1), 1-29 (2008)

26. Djolovic, I, Malkowsky, E: Matrix transformations and compact operators on some new mth-order difference sequences. Appl. Math. Comput. 198(2), 700-714 (2008)

27. Jarrah, AM, Malkowsky, E: Ordinary absolute and strong summability and matrix transformations. Filomat 17, 59-78 (2003)

28. Mursaleen, M, Noman, AK: Compactness by the Hausdorff measure of noncompactness. Nonlinear Anal. 73(8), $2541-2557(2010)$

\section{Submit your manuscript to a SpringerOpen ${ }^{\ominus}$ journal and benefit from:}

- Convenient online submission

Rigorous peer review

- Immediate publication on acceptance

- Open access: articles freely available online

- High visibility within the field

- Retaining the copyright to your article 